\title{
INNOVACIÓN, INVESTIGACIÓN Y DESARROLLO. ELEMENTOS EN EL SISTEMA DE INFORMACIÓN CIENTÍFICA Y TECNOLOGICA EN EL ESTADO DE BAJA CALIFORNIA
}

Margarita Ramírez-Ramírez ${ }^{1}$ Universidad Autónoma de Baja California. México maguiram@uabc.edu.mx, maguieram@yahoo.com.mx

Nora del Carmen Osuna-Millán: Universidad Autónoma de Baja California. México nosuna@hotmail.com, nosuna@gmail.com

Esperanza Manrique-Rojas: Universidad Autónoma de Baja California. México emanrique@uabc.edu.mx

Hilda Beatriz Ramírez-Moreno: Universidad Autónoma de Baja California. México ramirezmb@uabc.edu.mx

\section{RESUMEN}

Actualmente el avance en una sociedad depende del desarrollo de la ciencia y la tecnología en los diferentes ámbitos, vivimos en un periodo de rápida evolución, un momento en el que es necesario estudiar con mayor profundidad temas actuales como son: los cambios climáticos, los desastres naturales, el terrorismo, los conflictos nacionales e internacionales. Una actividad primordial para lograr eficientemente el desarrollo de una región es concentrar la información del conocimiento que demandan las empresas y la productividad relativa a ciencia y tecnología. Los indicadores de ciencia y tecnología son el principal soporte en la innovación, en México se han implementado políticas y acciones que apoyan el cambio tecnológico y la innovación en diferentes áreas. Una de las intenciones de Baja California es convertir a la ciencia, la tecnología y la innovación en los pilares del crecimiento económico. El presente trabajo pretende mostrar las características del desarrollo de una herramienta que proporciona información sobre las Instituciones de Educación

\footnotetext{
${ }^{1}$ Autor correspondiente

Margarita Ramírez-Ramírez: Universidad Autónoma de Baja California, Instituto Tecnológico de Tijuana. Tijuana, B.C. (México).

Correo: maguiram@uabc.edu.mx, maguieram@yahoo.com.mx
} 
Superior, los centros de investigación, la productividad de investigadores en el Estado de Baja California, con el objetivo de lograr unir esfuerzos en áreas de importancia para la región, promoviendo una relación de conocimiento, colaboración y apoyo entre instituciones, investigadores y gobierno.

PALABRAS CLAVE: Tecnologías - Información - Innovación - Investigación

\title{
INNOVATION, RESEARCH AND DEVELOPMENT, ELEMENTS OF THE SYSTEM OF INFORMATION SCIENCE AND TECHNOLOGY IN THE STATE OF BAJA CALIFORNIA
}

\begin{abstract}
Currently progress in a society depends on the development of science and technology in various fields, we live in a period of rapid evolution, a time that is necessary to study more deeply current topics such as: climate change, disasters natural, terrorism, national and international conflicts. A key activity for achieving efficient development of a region is to concentrate the knowledge that demand information and productivity firms on science and technology. Indicators of science and technology are the main support innovation, Mexico has implemented policies and actions to support technological change and innovation in different areas. One of the intentions of Baja California is to make science, technology and innovation in the pillars of economic growth. This paper aims to show the characteristics of the development of a tool that provides information on higher education institutions, research centers, the productivity of researchers in the State of Baja California, with the goal of uniting efforts in areas of importance to the region, promoting a relationship of knowledge, collaboration and support between institutions, researchers and government.
\end{abstract}

KEY WORDS: Technology - Information - Innovation - Research

\section{INTRODUCCIÓN}

Actualmente la sociedad depende en un porcentaje elevado del desarrollo de la ciencia y la tecnología, por lo tanto, el sector gobierno debe reforzar de una manera eficiente la toma de decisiones en lo relativo a las actividades científicas y tecnológicas del país, para ello es necesario concentrar a las entidades federativas en sistemas de información de investigación científica y tecnológica que permitan identificar las áreas principales del conocimiento que demandan las empresas y los grandes problemas regionales, nacionales e internacionales. 
Una de las intenciones de Baja California es convertir a la ciencia, la tecnología y la innovación en los pilares de su crecimiento económico. Por esto se ha desarrollado un sistema de información que permite la administración de cada uno de los puntos ya mencionados.

En la actualidad la innovación tecnológica es el inductor más importante del éxito competitivo en una organización, en una empresa y en la industria, al mismo tiempo que los indicadores de ciencia y tecnología son el principal soporte para la innovación en las empresas, los organismos y las comunidades de cualquier estado o país.

La presión para la innovación ha subido el nivel competitivo en las industrias y esto hace el éxito más complicado para las empresas, pero al mismo tiempo ha tenido un efecto positivo en la sociedad.

Este proceso de innovación fue desarrollado por los organismos encargados de impulsar y promover el desarrollo de la ciencia y la tecnología en el estado de Baja California, realizando esfuerzos por ofrecer elementos que apoyen a las empresas en el camino hacia la competitividad,

En atención a este interés y siguiendo el camino de la innovación, un equipo de investigadores-docentes de la Universidad Autónoma de Baja California, apoyados por el Consejo Nacional de Ciencia y Tecnología en México (CONACYT), el Gobierno del Estado de Baja California a través del Consejo de Ciencia y Tecnología (COCYT) diseñó e implementó un sistema de información SIEDCyT el cual permite integrar y dar seguimiento a las acciones realizadas en lo relativo a ciencia y tecnología en la región por los investigadores reconocidos en las distintas instituciones y centros de investigación.

Vivimos en un periodo de rápida evolución, un momento en el que es necesario estudiar con mayor profundidad y exactitud, los temas actuales como lo son: los cambios climáticos, los desastres naturales, el terrorismo, los conflictos nacionales e internacionales en los países. Pero también es importante, el que estos avances y/o estudios, que son realizados por académicos, investigadores e instituciones sean difundidos a la sociedad, por y para quien fueron realizados.

A continuación se detallan las etapas y procesos que se llevaron a cabo en la gestión del proyecto. 


\section{METODOLOGÍA}

\section{Gestión del Proyecto}

La gestión de un proyecto, implica el conjunto de trámites que se llevan a cabo para resolver un problema, asunto o concretar un proyecto, está también conlleva la administración del proyecto. En este apartado se describirán las fases de desarrollo del proyecto que se llevaron a cabo.

\subsection{Fase Planeación e Integración}

Como fase inicial se realizaron las actividades relativas al trámite de recursos, se organizó y ejecuto las acciones necesarias para integrar el grupo de investigadores y participantes en el proyecto para estandarizar conocimientos e información relativa al proyecto.

Una de las tareas primordiales para realizar una gestión adecuada fue la investigación preliminar, en la cual se obtuvo una perspectiva clara de los requerimientos del proyecto.

Dentro de las actividades primordiales se llevaron a cabo: La identificación de políticas y reglas permitidas para la gestión de la información de los objetos de estudio. La implementación de un sistema que apoye la recopilación de información y documentación.

Una vez completada la fase de inicio, se dio seguimiento a la fase de elaboración, en la cual las actividades se orientan en el desarrollo de las políticas y reglas definidas para la gestión de la información, se llevaron a cabo las actividades relativas al análisis y diseño del sistema, se desarrollaron las estrategias base para la implementación del sistema.

En forma paralela al análisis del sistema se trabajó en las actividades en relación a la recopilación de la información.

\subsection{Fase de inicio}

a) Recopilación de la información requerida por los usuarios del sistema

b) Diseño de cuestionario para recopilación de información

c) Proceso de validación del cuestionario.

d) Diseño de entrevistas estructuradas.

e) Recopilación de la información en cada una de las instituciones 


\subsection{Fase Diseño}

Una vez obtenidos los requerimientos de información y aprobada la propuesta, se inició la labor de diseño en sus distintas unidades:
a) Procesos, funciones o métodos
b) Bases de datos
c) Entradas y salidas de datos
d) Interfaces de usuarios y programas de aplicación

\subsection{Fase de Desarrollo}

a) La construcción del sistema de información vía Web, aplicando técnicas de ingeniería de usabilidad de sitios Web

b) Diseño de imagen exterior del sistema

c) La elaboración de manuales de usuario y manuales técnicos

\subsection{Fase de Implementación}

Concluida la etapa del diseño y desarrollo, se llevaron a cabo las siguientes actividades:
a) Pruebas del sistema en el entorno real
b) Capacitación de usuarios
c) Integración de la base de datos

\section{ANÁLISIS Y DISCUSIÓN}

\subsection{Innovación}

Una fuente de innovación procede de las instituciones de investigación pública como son las universidades, laboratorios públicos e incubadoras. Estas innovaciones generadas por este tipo de instituciones permiten a las empresas desarrollar productos o servicios que tal vez no se hubiesen generado.

La innovación y el cambio técnico no son procesos lineales ni características de una empresa, sino el resultado de un ambiente, un entorno que promueve espacios con capacidad para generar, adoptar y difundir innovaciones, conocidos como espacios emergentes, regiones que aprenden $\mathrm{o}$, entorno innovador.

Un territorio innovador es un sistema productivo vinculado a una o varias actividades, en el que una buena parte de las empresas existentes realizan esfuerzos en el plano de la innovación tecnológica, incorporando mejoras en los diferentes procesos asociados a su cadena de valor y en los productos que ofrece. 
El concepto de medio innovador se basa en la tesis de que la innovación no se realiza en forma individual, sino colectiva, en estrecha relación con la presencia de un clima empresarial, social e instituciones favorables.

El rol del gobierno local en la implementación de medios innovadores, se concreta en tres acciones de conectividad:

a) en el nivel social, facilitando la interacción entre los demás actores participantes del proceso de innovación y desarrollo: empresas, universidades y centros de investigación, y organizaciones comunitarias;

b) en el nivel técnico, desarrollando un medio innovador en presencia de buenas telecomunicaciones y un excelente servicio de transporte,

c) en el tercero, de conectividad asegurando las interacciones entre el medio innovador, la ciudad y la región.

Atendiendo las intenciones de concentrar la información de todas las instituciones educativas, centros de investigación, recursos humanos e infraestructura relacionada con la generación de Ciencia y tecnología, se desarrolló e implementó una herramienta, un Sistema de información administrado por un sitio web el cual almacena, procesa y presenta información relevante de Ciencia y Tecnología, con la intención de apoyar en la toma de decisiones, así como la colaboración de instituciones que estén trabajando o hayan trabajado en proyectos similares.

\subsection{SIEDCyT}

El SIEDCyT (Sistema estatal de Información y documentación científica y tecnológica de recursos humanos, materiales, de organización y financieros), proyecto que surgió en respuesta a la convocatoria de fondos mixtos de CONACYT y gobierno del estado. SIEDCyT es una plataforma que integra las capacidades e indicadores de la productividad científica, tecnológica y de innovación en el estado, desarrollado para mostrar a través de un portal accedido vía web, las capacidades de investigación y desarrollo tecnológico, el presente trabajo pretende contar las experiencias vividas en el desarrollo de este proyecto. A continuación se muestran pantallas del sistema en funcionamiento. 


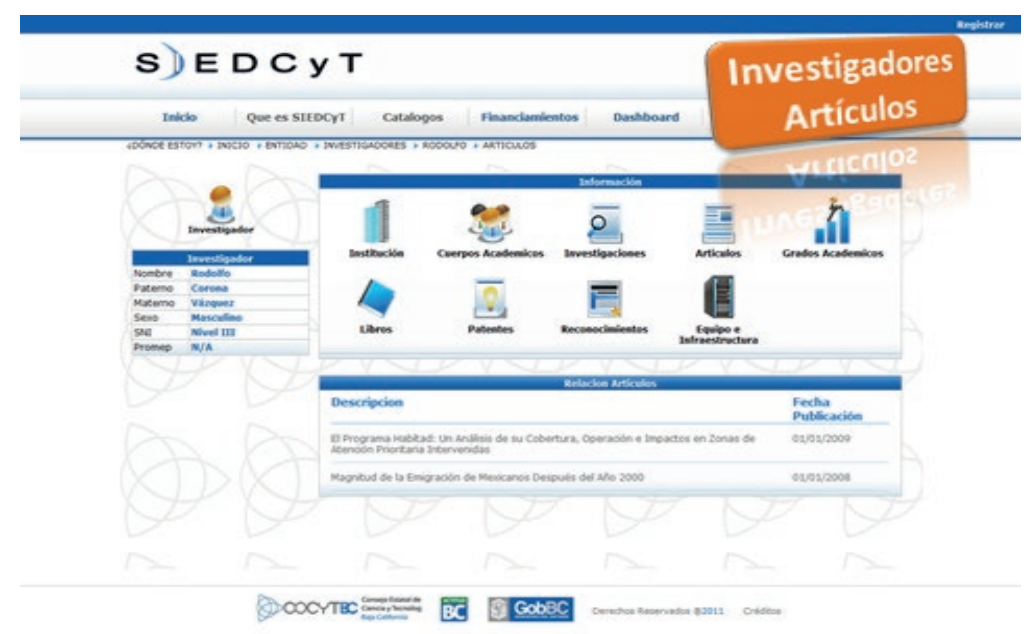

Figura 1 .Pantalla Sistema SIEDCyT Artículos por Investigador. Fuente SIEDCyT

Pantalla en la que se observa la información general del investigador y los artículos que ha producido.

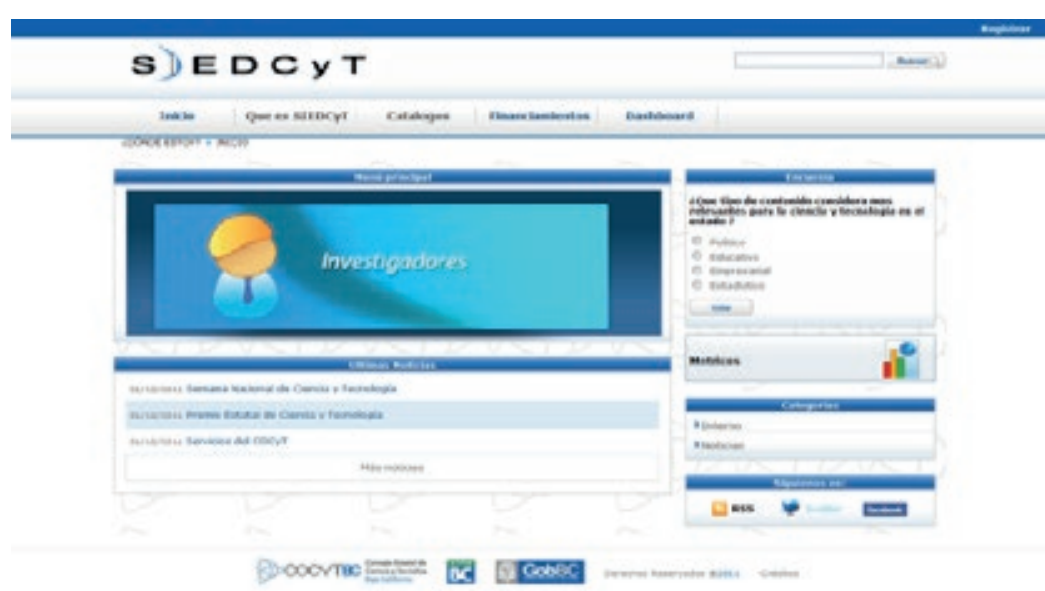

Figura 2. Pantalla Sistema SIEDCyT Investigadores Fuente SIEDCyT

Pantalla en el que se registra la información de los investigadores, en el lado derecho se observa el resultado de las respuestas a preguntas de opinión, que sean planteadas por los administradores del sistema. 


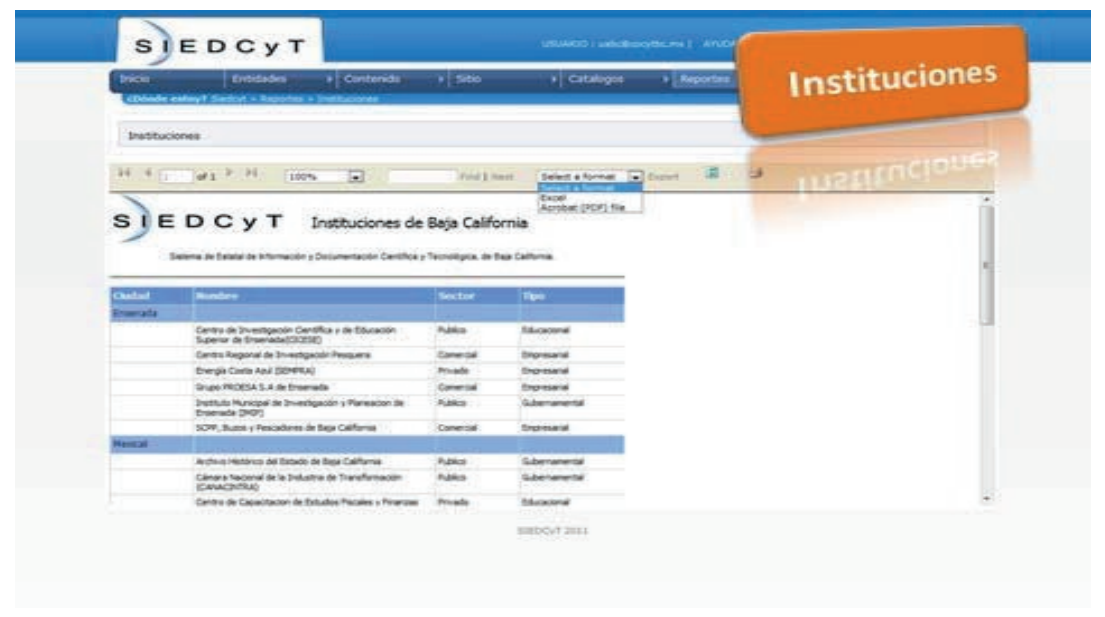

Figura 3. Pantalla Sistema SIEDCyT Instituciones. Fuente propia

Pantalla en la que es posible realizar el registro y presentación de las instituciones almacenadas en el sistema.

\subsection{Módulos de sistema SIEDCyT}

Al sistema SIEDCyT lo integran los siguientes módulos:

a) Módulo de registro de instituciones y centros de Investigación, investigadores y grupos de investigación.

b) Módulo de creación y mantenimiento de los catálogos de información de: Infraestructura y equipamiento destinados a la Ciencia y Tecnología.

c) Directorio de instituciones y centros de investigación.

d) Listado de Investigadores pertenecientes al SNI, en el estado de Baja California.

\subsection{Dashboard}

Una de las fortalezas de este sistema es la posibilidad de contar con un dashboard, el cual es un panel de presentación de indicadores que reflejen la situación actual o corriente de ciertos atributos del sistema. 


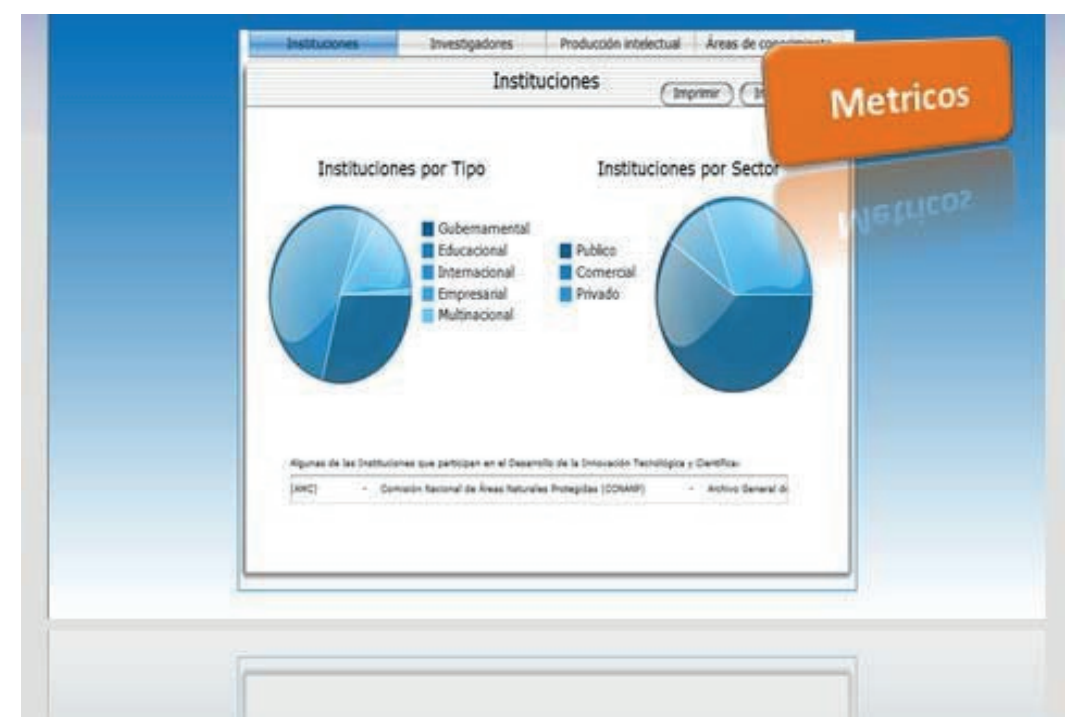

Figura 4. Pantalla Dashboard Sistema SIEDCyT: Fuente SIEDCyT

En la figura anterior se muestra una imagen del resultado de una consulta en el Dashboard.

\subsection{Almacén de datos}

En el sistema también se integró el diseño de un almacén de datos del SIEDCyT, el cual sirve de soporte para la toma de decisiones mediante herramientas de análisis.

El almacén de datos (Datamart) provee información a nivel estatal sobre ciencia y tecnología para planear, crear estrategias y tomar decisiones sobre la asignación de recursos en el estado. Proporcionar métricos exactos sobre el desempeño y productividad científica y tecnológica del estado. Evitar degradar el desempeño del sitio web SIEDCyT al momento de obtener reportes o generar nuevas preguntas

Crear un ambiente amigable de reportes, en el cual los usuarios puedan generar y mantener sus propios reportes, sin asistencia alguna del personal de tecnologías de información.

A continuación se presentan algunas pantallas de los datos buscados en los archivos de datos (carga del modelo dimensional de grados adquiridos). 


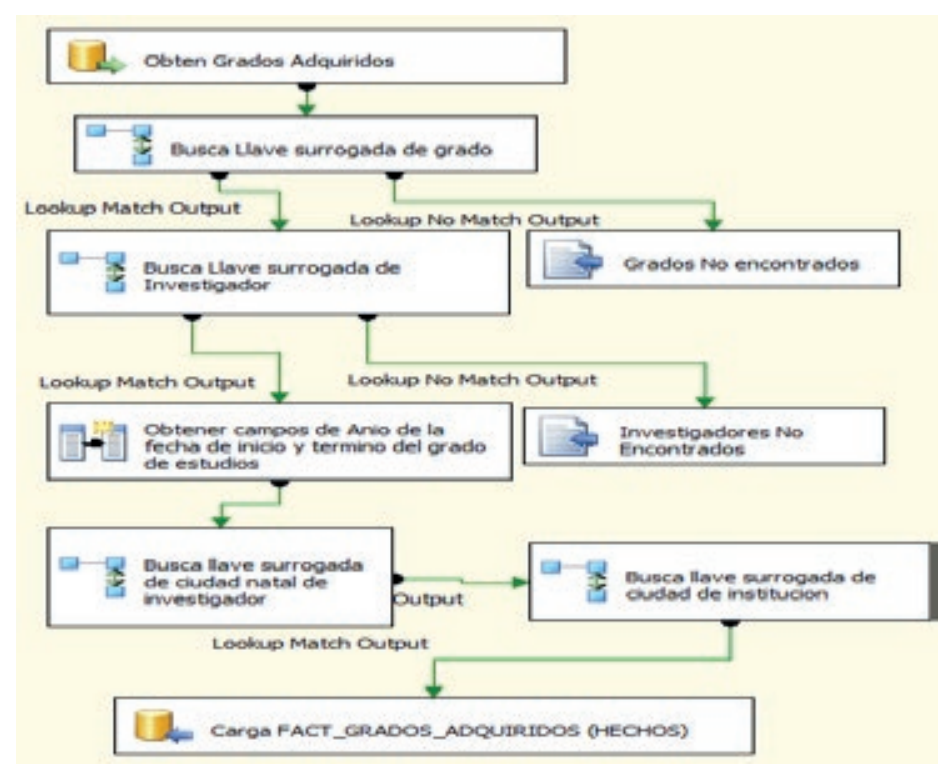

Figura 5. Carga de tabla de hechos grados adquiridos. Fuente Almacén de datos SIEDCyT

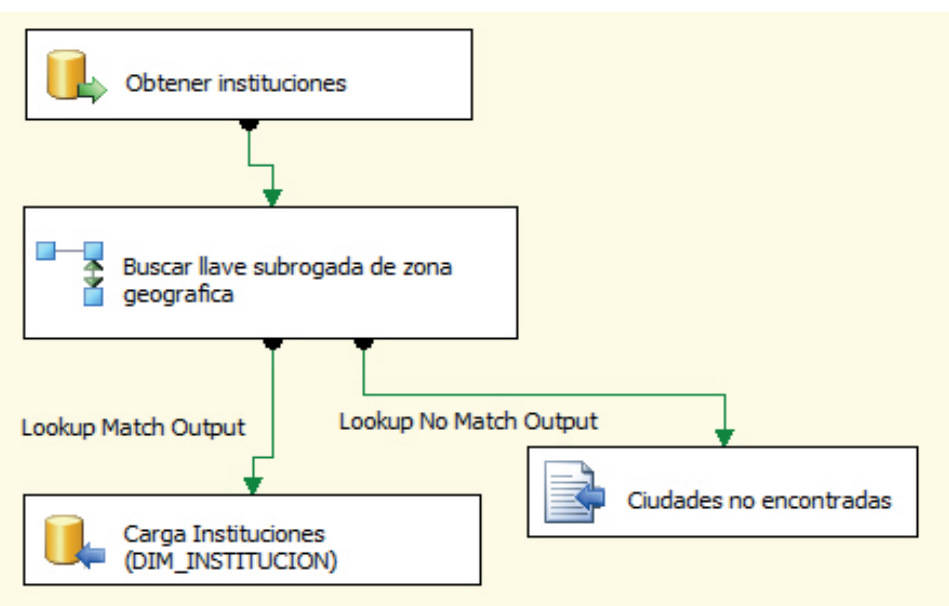

Figura 6. Carga de dimensión instituciones. Fuente Almacén de datos SIEDCyT

\subsection{Investigación}

Para la realización de este proyecto fue necesario y muy relevante la integración de un equipo de trabajo multidisciplinario, en el que se integraron los esfuerzos de investigadores-docentes del área de Informática, expertos en el área de estadística, maestros en desarrollo humano, ingenieros expertos en desarrollo de software, estudiantes de la Maestría en Tecnologías de la Información y la Comunicación, estudiantes de la Licenciatura en Informática, esta multidisciplinariedad y la experiencia de trabajar con un grupo conformado por estudiantes de diferentes grados, maestros e investigadores aporto al proyecto elementos valiosos que permitieron alcanzar las metas y fortalecieron el trabajo. 
El objetivo primordial del proyecto fue generar un sistema de información que ofrezca a los interesados información relativa a la productividad que en ciencia y tecnología se genera en el estado. De igual modo ofrece la posibilidad de registrar un inventario que sirva de apoyo en la toma de decisiones a los organismos correspondientes.

La conformación del inventario fue una de las actividades más enriquecedora del proyecto, ya que se tuvo como universo inicial de la investigación a los investigadores del estado miembros del sistema nacional de investigadores (SNI), 508 investigadores registrados.

Se aplicaron cuestionarios y realizaron entrevistas personalizadas en la mayoría de los casos, los datos obtenidos se integraron en la base de datos del sistema, estos generan información que permiten obtener indicadores de Ciencia y tecnología.

\subsection{Cuestionario}

El cuestionario aplicado a los investigadores permitió obtener información, misma que puede ser visualizada desde las herramientas del sistema.

Los apartados o bloques de preguntas en el cuestionario, son las siguientes:

a) Perfil de Investigador

b) Adquisición del conocimiento

c) Intercambio de conocimiento

d) Creación de nuevo conocimiento

e) Difusión del conocimiento

f) Aplicación comercial del conocimiento

g) Diagnóstico

Se aplicaron 290 cuestionarios a los investigadores distribuidos en diferentes instituciones de educación superior y centros de investigación distribuidos en los municipios de Tijuana, Ensenada y Mexicali.

\subsection{Análisis de los datos}

Se calculó la muestra para determinar el número mínimo requerido para la validez del trabajo de investigación; Sin embargo la cantidad de entrevistados supero el tamaño de la muestras obtenido en el cálculo, a continuación se describen los valores de determinación de la muestra. 


\section{Determinación de la muestra:}

Tabla 1. Determinación de la muestra. Fuente: Elaboración propia

\begin{tabular}{|cccc||}
\hline Nivel de Confianza & Valor de $\mathrm{z}$ & \% de Error & Valor de la proporción \\
$99 \%$ & 2.58 & $\mathrm{E}=0.10$ & $\mathrm{p}=.5$ \\
\hline
\end{tabular}

Tabla 2. Tamaño de la muestra. Fuente: Elaboración propia

Población $\quad \mathrm{N} \quad \mathrm{n}$

\begin{tabular}{|c|c|c|}
\hline ENSENADA & $\mathbf{2 8 4}$ & $\mathbf{1 0 5}$ \\
\hline CICESE & 135 & 75 \\
\hline UABC & 83 & 56 \\
\hline UNAM & 64 & 46 \\
\hline IT ENS & 1 & 1 \\
\hline INST & 1 & 1 \\
\hline SANIDAD & & \\
\hline TIIUANA & $\mathbf{1 3 7}$ & $\mathbf{7 5}$ \\
\hline ITT & 19 & 17 \\
\hline COLEF & 55 & 40 \\
\hline IPN & 10 & 9 \\
\hline UABC & 53 & 42 \\
\hline MEXICALI & $\mathbf{8 3}$ & $\mathbf{3 6}$ \\
\hline COLEF & 2 & 2 \\
\hline UABC & 77 & 53 \\
\hline UNIV. POL & 4 & 4 \\
\hline
\end{tabular}

A continuación se muestra información obtenida en el trabajo de campo de la investigación. 
Información del gobierno del estado de Baja California:

Número de investigadores del Sistema Nacional de Investigadores en los años del 2002 al 2010.

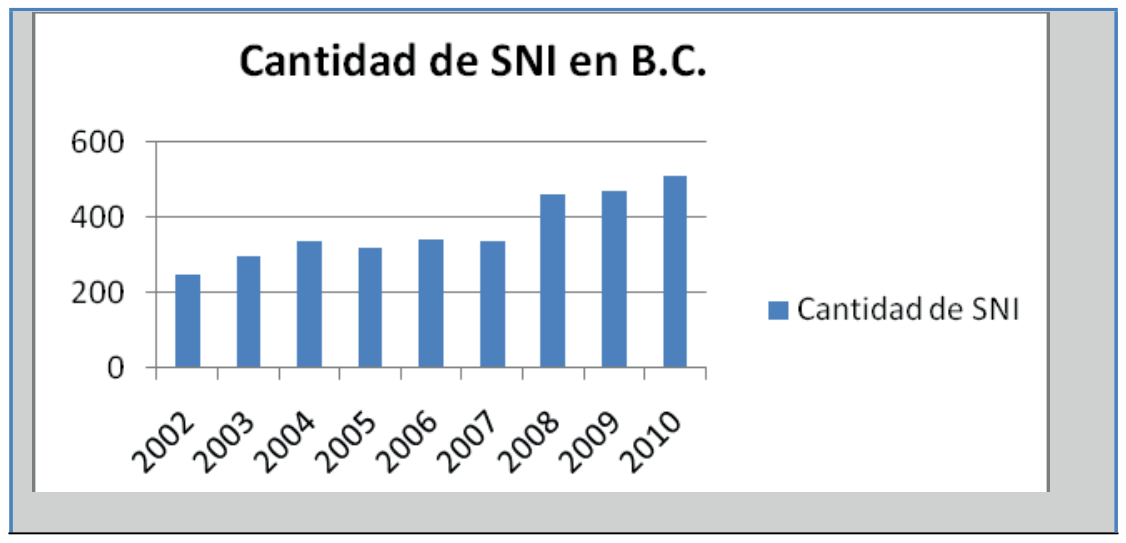

Figura 7. Cantidad de SNI en Baja California. Fuente: Elaboración propia

Tabla 3. Número de investigadores del SNI por niveles, período 2002-2010.Fuente: Elaboración propia

\begin{tabular}{|l|c|c|c|c|l|l|}
\hline AÑO & Candidato & Nivel I & $\begin{array}{c}\text { Nivel } \\
\text { II }\end{array}$ & $\begin{array}{c}\text { Nivel } \\
\text { III }\end{array}$ & Emérito & Total \\
\hline 2002 & 31 & 157 & 47 & 12 & & 247 \\
\hline 2003 & 41 & 185 & 52 & 17 & & 295 \\
\hline 2004 & 49 & 203 & 60 & 22 & & 334 \\
\hline 2005 & 50 & 181 & 64 & 21 & & 316 \\
\hline 2006 & 58 & 195 & 64 & 21 & & 338 \\
\hline 2007 & 40 & 197 & 72 & 25 & & 334 \\
\hline 2008 & 58 & 264 & 111 & 25 & & 458 \\
\hline 2009 & 66 & 253 & 115 & 33 & & 467 \\
\hline 2010 & 83 & 263 & 123 & 38 & 1 & 508 \\
\hline
\end{tabular}




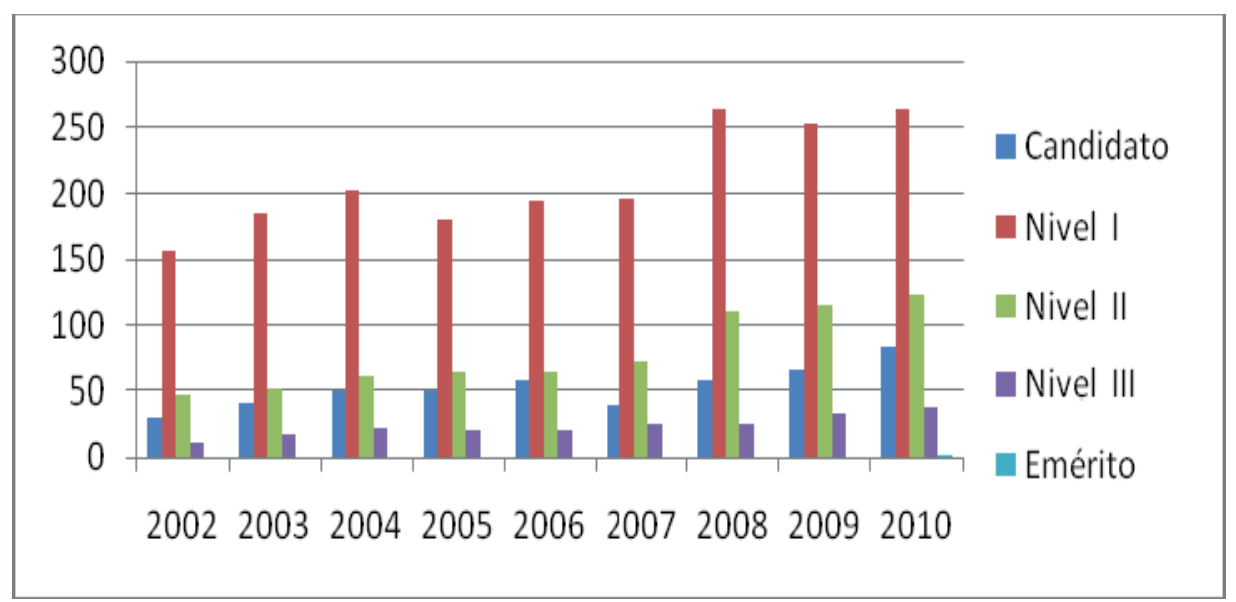

Figura 8. Número de investigadores del SNI por niveles. Fuente: Elaboración propia

Tabla 4. Número de investigadores del SNI por Áreas de conocimiento. Fuente: Elaboración propia

\begin{tabular}{|c|c|c|c|c|c|c|c|c|c|}
\hline Área académica & $\mathbf{2 0 0 2}$ & $\mathbf{2 0 0 3}$ & $\mathbf{2 0 0 4}$ & $\mathbf{2 0 0 5}$ & $\mathbf{2 0 0 6}$ & $\mathbf{2 0 0 7}$ & $\mathbf{2 0 0 8}$ & $\mathbf{2 0 0 9}$ & $\mathbf{2 0 1 0}$ \\
\hline $\begin{array}{c}\text { I. Físico } \\
\text { Matemático }\end{array}$ & 112 & 133 & 147 & 131 & $14 /$ & $14 /$ & $18 /$ & 177 & 177 \\
\hline $\begin{array}{c}\text { II. Biología y } \\
\text { Química }\end{array}$ & 40 & 52 & 55 & 47 & 50 & 45 & 52 & 62 & 54 \\
\hline $\begin{array}{c}\text { III. Medicina y } \\
\text { Ciencias de salud }\end{array}$ & 0 & & 3 & 5 & 5 & 5 & 5 & 4 & 3 \\
\hline $\begin{array}{c}\text { IV. Humanidades } \\
\text { y Ciencias de la } \\
\text { Conducta }\end{array}$ & 11 & 15 & 17 & 15 & 16 & 17 & 26 & 30 & 35 \\
\hline $\begin{array}{c}\text { V. Ciencias } \\
\text { Sociales }\end{array}$ & 35 & 40 & 42 & 39 & 42 & 44 & 89 & 91 & 109 \\
\hline $\begin{array}{c}\text { VI. Biotecnología } \\
\text { y Ciencias } \\
\text { Agropecuarias }\end{array}$ & 12 & 15 & 24 & 30 & 31 & 30 & 37 & 36 & 43 \\
\hline $\begin{array}{c}\text { VII. Ingenierías } \\
\text { IIngen }\end{array}$ & 31 & 40 & 46 & 49 & 54 & 53 & 69 & 67 & 87 \\
\hline
\end{tabular}




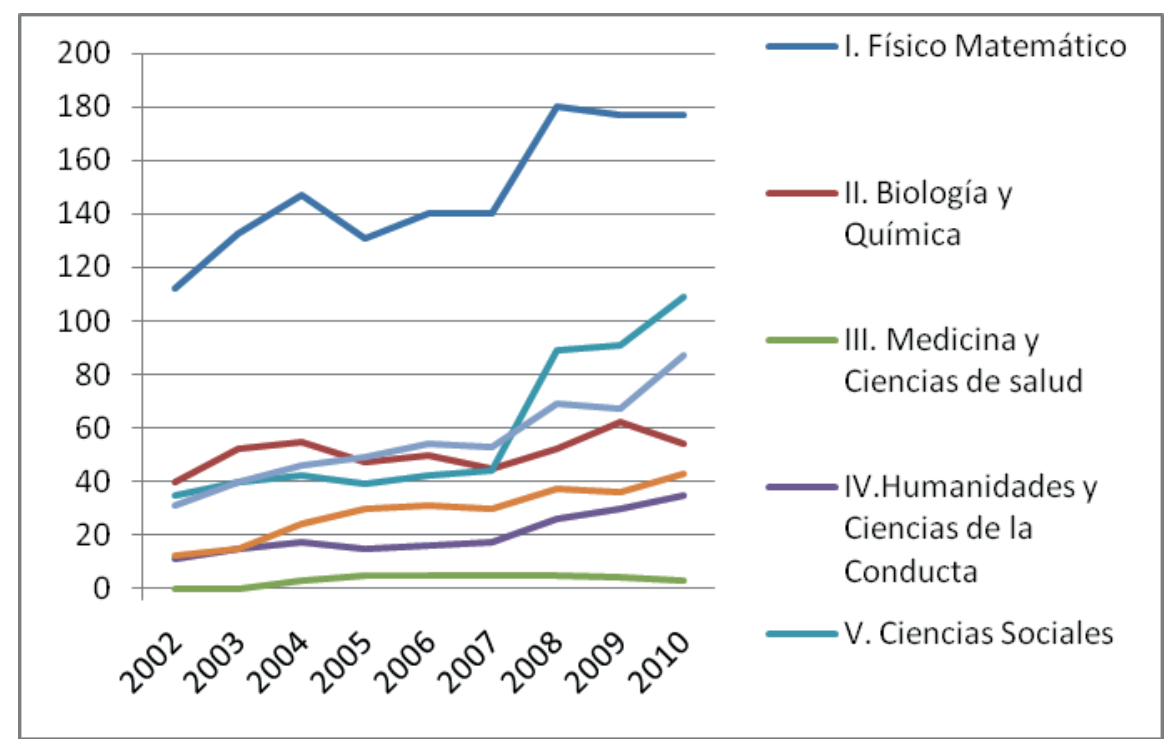

Figura 9. Número de investigadores del SNI por Áreas de conocimiento. Fuente: Elaboración propia

\section{CONCLUSIONES}

El desarrollo de la Ciencia, los desarrollos tecnológicos y la innovación, son elementos primordiales en el desarrollo de una región, no es posible concebir una cultura sobresaliente, si no se tiene a la par avances científicos, innovación y una actitud competitiva y comprometida con la calidad en los sectores académicos, de investigación e industriales.

Las estrategias que impulsan la difusión de la ciencia y la tecnología por las instituciones gubernamentales, en conjunto con instituciones académicas y de investigación pueden ser un factor determinante para el logro de estos objetivos y metas.

El desarrollo de sistemas como el que aquí se presenta tiene como objetico mejorar los medios y canales de acceso a la información, para maximizar la aplicación de los estudios e investigaciones que se llevan a cabo en beneficio del engrandecimiento y mejora del estado (Plascencia, 2010). La presentación de este trabajo conjunta el resultado del esfuerzo de investigadores, especialistas del área de desarrollo de tecnologías, académicos y estudiantes de las Tecnologías de la información y la comunicación, todos ellos con la intención de avanzar en las metas definidas en el área de Ciencia y Tecnología en el estado de Baja California. 


\section{REFERENCIAS}

Bernal Torres, C. A. (2006). Metodología de la investigación: para administración, economía, humanidades y ciencias sociales. México: Pearson Educación.

Cabrero Mendoza, E. (2006). El diseño institucional de la política de ciencia y tecnología en México. México: Universidad Nacional Autónoma de México.

Cohen Karen, D. (2005). Sistemas de información para los negocios: un enfoque para la toma de decisiones. México: McGraw-Hill.

Gido Jack, J. C. (2008). Administración exitosa de proyectos. México: CENGAGE Learning

Hernández Sampieri, R. (2007). Fundamentos de metodología de la investigación. México: McGraw-Hill.

Kendall Kenneth, E. (2005). Análisis y diseño de sistemas. México: Pearson educación.

López García, X. (2006). Sistemas digitales de información. . México: Pearson Prentice Hall.

O'brien James, A. (2006). Sistemas de información gerencial. . México: McGraw-Hill. Plascencia

López, I. (2010). Actitudes públicas acerca de la ciencia en Baja California. México.

Rodríguez Durán, A. (2002). Ciencia, tecnología y ambiente. . México: International Thomson.

Salomon, J. J. (1996). Una búsqueda incierta: ciencia, tecnología y desarrollo, Editorial de la Universidad de las Naciones Unidas. México: Centro de Investigación y Docencia Económicas: FCE.

Takayanagui, A. D. (2000). La universidad del futuro: relaciones entre la educación superior, la ciencia y la tecnología. México: UNAM.

Tamayo Tamayo, M. (2007). El proceso de la investigación científica: incluye evaluación y administración de proyectos de investigación. . México: Limusa.

Schilling, A. M. (2008). Dirección estratégica de la innovación tecnológica. España: McGraw Hill, 
Uscanga, C. (2008). Las políticas para el desarrollo de la ciencia y tecnología en Japón. México: UNAM.

CONACYT. (2006). Ciencia y tecnología para la competitividad, Fondo de cultura económica. México

CONACYT. (2006). Informe general del estado de la ciencia y la tecnología. México

\section{Margarita Ramírez Ramírez}

Grado de maestra en Desarrollo Humano, por la Universidad Iberoamericana del Noroeste, y la maestría en Ciencias en Ciencias de la Computación por Instituto Tecnológico de Tijuana. Se desempeño como Subdirectora Académica de la Facultad de Contaduría y Administración. Fue presidenta de Academia Ciencias Administrativas, en la UABC, período 2007-2009. Ha sido profesora en la Facultad de Contaduría y Administración, de la Universidad Autónoma de Baja California, campus Tijuana desde 1990 a la fecha, así como profesora en el Instituto Tecnológico de Tijuana, a partir del año 1991. Es miembro del cuerpo fundador de Academia Nacional ANFECA, así como Certificación de Informática Administrativa por ANFECA, cuenta con Perfil PROMEP. Actualmente coordinadora de la Maestría en Tecnologías de la Información y la Comunicación en la Facultad de Contaduría y Administración.

\section{Nora del Carmen Osuna Millán}

Estudio la Lic. En Sistemas Computacionales en la Universidad de Occidente, La Maestría en Ciencias en Ciencias Computacionales, por Instituto Tecnológico de Tijuana, Cuenta con la Certificación por ANFECA como Licenciada en Informática Administrativa, cuenta con el perfil PROMEP, Se ha desempeñado como docente en la Licenciatura en Informática y la Maestría en Tecnologías de la Información y la Comunicación en la Facultad de Contaduría y Administración Tijuana ha tomado cursos como: Curso de Inteligencia Artificial, Curso de Sistemas Basados en Conocimiento, implementing a Microsoft SQL Server 2005 Database, Developing Microsoft ASP.NET web Applications Using Visual Studio .NET entre otros.

Actualmente es titular del órgano interno de control en Exportadora de sal S.A de C.V. en B.C.S.

\section{Esperanza Manrique Rojas}

Obtuvo la maestría en Ciencias en Ciencias de la Computación por Instituto Tecnológico de Tijuana, ha sido profesora en la Facultad de Contaduría y Administración, de la Universidad Autónoma de Baja California, campus Tijuana desde 1992 a la fecha, actualmente es miembro del cuerpo académico Sistemas de Información y Gestión Empresarial, como Certificación Académica por ANFECA, cuenta con Perfil PROMEP, actualmente es Coordinadora del proyecto Adultos en Plenitud Aprendiendo nuevas Tecnologías, así como coordinadora del programa de acreditación v aseóriramiento de Prooramas académicns 


\section{Hilda Beatriz Ramírez Moreno}

Obtuvo su grado de maestra en Ciencias en Ciencias de la Computación por el Instituto Tecnológico de Tijuana, ha impartido clases en la Facultad de Contaduría y Administración, de la Universidad Autónoma de Baja California, campus Tijuana durante 12 años. Actualmente es miembro del cuerpo académico Sistemas de Información y Gestión Empresarial, cuenta con Certificación Académica por ANFECA y Perfil PROMEP, actualmente es Coordinadora del proyecto Adultos en Plenitud Aprendiendo nuevas Tecnologías, así como Coordinadora de Área de Sistemas de Información de la carrera de Lic. en Informática. 